\title{
Suporte familiar e sintomatologia depressiva e ansiosa em mulheres com obesidade
}

\author{
Nathália Gomes da Silva \\ José Roniere Morais Batista \\ Melyssa Kellyane Cavalcanti Galdino \\ Sibelle Maria Martins de Barros
}

\section{RESUMO}

O objetivo deste estudo consistiu em descrever a correlação do grau de percepção do suporte familiar com o nível de sintomatologia depressiva e ansiosa em pacientes com obesidade. A amostra foi composta por pacientes do sexo feminino $(\mathrm{N}=39)$, usuárias do Sistema Único de Saúde (SUS), com idade média de 41 anos (DP = 10,74). As participantes responderam ao Inventário de Depressão de Beck (BDI), ao Inventário de Ansiedade de Beck (BAI) e ao Inventário de Percepção do Suporte Familiar (IPSF). Os resultados indicaram correlações negativas significativas entre o IPSF com o BDI e o BAl, indicando que, quanto maior a percepção do suporte familiar recebido, menores são os escores de sintomas depressivos e ansiosos. Correlações entre o BDI e o BAI também foram encontradas, apontando que quanto maior o grau de depressão, maior o nível de ansiedade. De maneira geral, os estudos indicam a efetiva importância do suporte familiar que se percebido como adequado pelo indivíduo, pode se apresentar como um fator de proteção no processo saúde-doença.

Palavras-chave: obesidade; depressão; ansiedade; suporte familiar.

\section{ABSTRACT}

\section{Family support, depressive and anxious symptomatology in obese women}

The aim of this study was to verify the correlation of perception of family support and depressive and anxious symptoms in patients under treatment for obesity. The sample was composed by female patients $(\mathrm{N}=39)$, users of the Unified Health System (SUS), with a mean age of 41.00 years $(S D=10.74)$. The participants answered the Beck Depression Inventory (BDI), the Beck anxiety Inventory (BAI) and the Inventory of Perception of Family Support (IPSF). The results show significant negative correlations among the IPSF andthe BDI and the $\mathrm{BAI}$, indicating that the higher the perception of family support received, the lower the scores of anxious and depressive symptoms. Correlations between the BDI and BAI were also found, pointing that the higher the degree of depression, the higher was the patient's anxiety level. In general, studies show the importance of effective family support, that if perceived as appropriate by the individual, may be a protective factor in the health-disease process.

Keywords: obesity; depression; anxiety; family support.

A obesidade é uma doença crônica, de etiologia multifatorial, caracterizada principalmente pelo acúmulo excessivo de tecido adiposo no organismo. É associada a comorbidades, como diabetes, doenças cardiovasculares, hipertensão, depressão e comportamentos de esquiva social, que prejudicam a qualidade de vida do indivíduo (Ades \& Kerbauy, 2002). A prevalência crescente da obesidade representa um dos principais desafios de saúde pública no início deste século (Coutinho, 2007).

No Brasil, estima-se que o índice de sobrepeso tenha atingido mais da metade da população. Dados divulgados pelo Ministério da Saúde apontavam que, em 2006, 42,7\% da popula-

\section{Sobre os Autores}

N.G.S.

orcid.org/0000-0001-9330-3059

Universidade Estadual da

Paraíba (UEPB) - Campina

Grande, PB

nathaliagomes@outlook.com

\section{J.R.M.B}

orcid.org/0000-0002-9055-7544

Universidade Federal de

Campina Grande (UFCG) -

Campina Grande, PB

roniere@gmail.com

M.K.C.G.

orcid.org/0000-0001-7180-3458

Universidade Estadual da

Paraíba (UEPB) - Campina Grande, PB

melyssa_cavalcanti@hotmail.co $\mathrm{m}$

\section{S.M.M.B}

orcid.org/0000-0003-0933-5475 Universidade Federal da Paraíba (UFPB) - Campina Grande, PB sibellebarros@yahoo.com.br

\section{Direitos Autorais}

Este é um artigo de acesso aberto e pode ser reproduzido livremente, distribuído,

transmitido ou modificado, por qualquer pessoa desde que usado sem fins comerciais. 0 trabalho é disponibilizado sob a licença Creative Commons CCBY-NC. 
ção estava acima do peso, e, em 2011, esse número avançou para $48,5 \%$. Em pesquisa lançada em 2015 , a frequência de excesso de peso alcançou $52,5 \%$, sendo maior entre homens $(56,5 \%)$ do que entre mulheres $(49,1 \%)$ (Ministério da Saúde, Secretaria de Vigilância em Saúde, Departamento de Vigilância de Doenças e Agravos não Transmissíveis e Promoção da Saúde, 2015).

Existem vários métodos para dimensionar o grau de obesidade de um indivíduo, a saber: Índice de Massa Corporal (IMC), tabelas de peso e altura, relação cintura-quadril, circunferência abdominal, exames laboratoriais, entre outros. Entretanto, o IMC é o parâmetro utilizado com maior frequência em nível populacional e na prática clínica, por se tratar de um cálculo simples, que apresenta boa correlação com a adiposidade corporal. Este índice é calculado pela relação entre peso e estatura, sendo expresso $\mathrm{em} \mathrm{Kg} / \mathrm{m}^{2}$, e sugere as seguintes classificações: 1) entre 25 e $29,9 \mathrm{Kg} / \mathrm{m}^{2}$ = sobrepeso; 2) entre 30 e $39,9 \mathrm{Kg} / \mathrm{m}^{2}$ = obesidade; e c) maior que $40,0 \mathrm{Kg} / \mathrm{m}^{2}=$ obesidade mórbida (Associação Brasileira para o Estudo da Obesidade e da Síndrome Metabólica - ABESO, 2002).

A indicação ao tratamento está associada à avaliação multidisciplinar, composta por endocrinologistas, nutricionistas, cardiologistas, pneumologistas, assistentes sociais, psiquiatras e psicólogos. O acompanhamento psicológico é considerado essencial, uma vez que as pessoas obesas que procuram tratamento demonstram a prevalência de sintomas depressivos, ansiosos e alterações do comportamento alimentar (Gold, Oppenheim \& Frost-Pineda, 2006).

Conforme o Conselho Latino-Americano de Obesidade, o paciente obeso manifesta grande sofrimento psicológico decorrente do preconceito pelo seu excesso de peso e pelas características do seu comportamento alimentar (Coutinho, 1999). Comumente, tal sofrimento é visto como consequência da obesidade, no entanto, conflitos e problemas psicológicos podem preceder o desenvolvimento dessa condição; na realidade, pessoas obesas evidenciam aspectos emocionais e psicológicos que são reconhecidos como potenciais causas, consequências ou retroalimentadores da obesidade (Vasques, Martins, \& Azevedo, 2004). Desse modo, é fundamental novas possibilidades de pesquisa que abordem a obesidade através de uma perspectiva que considere a etiologia, variáveis cognitivas, emocionais e ambientais idiossincráticas (como hábitos alimentares e familiares, por exemplo).

Entre os problemas psiquiátricos, a depressão é o indicador de maior prevalência em casos de obesidade grave. Já a ansiedade é o segundo indicador de maior prevalência nesta população (Vargas, 2012). No tocante à relação entre obesidade e depressão, Saueressig e Berleze (2010), encontraram como resultado depressão leve a moderada em $20,5 \%$ da amostra estudada, depressão moderada a grave em $14,4 \%$ e depressão grave em $2,6 \%$ da referida amostra. Correia e Afonso (2012) verificaram que a sintomatologia depressiva afeta transversalmente a população universitária; contudo, jovens do sexo feminino com excesso de peso são mais afetadas por esta condição.

Matos et al. (2002), investigaram aspectos psicológicos em pacientes, de ambos os sexos, com idade entre 18 e 65 anos, diagnosticados com obesidade grau III que procuraram tratamento no ambulatório de obesidade da Universidade Federal de São Paulo (UNIFESP). Os autores verificaram a ocorrência de episódios de compulsão alimentar periódica em $54 \%$ da amostra. Foram verificados, também, sintomas depressivos em $100 \%$ dos participantes, sendo que, destes, $84 \%$ apresentavam sintomatologia grave. Já a frequência de ansiedade foi $70 \%$ como traço e $54 \%$ como estado. A preocupação com a imagem corporal esteve presente em $76 \%$ dos pacientes.

Emoções negativas e sofrimento psicológico também podem estar associados a um ambiente familiar pouco apoiador. Os filhos estão propensos a apresentar características ineficazes de enfrentamento, conforme os pais passam por situações estressoras e manifestam características semeIhantes de enfrentamento. Este fato pode acarretar prejuízos significativos, como rebaixamento da autoestima e do autoconceito ou o desenvolvimento de ansiedade e sintomas depressivos (Baptista, Cardoso, \& Gomes, 2012).

Moraes, Almeida e Sousa (2013) investigaram a percepção de obesos com depressão sobre os fatores envolvidos na manutenção da própria obesidade em 68 usuários obesos, sendo 21 identificados com escore significativo para depressão. Foi encontrada a categoria ambiente familiar como um dos reforçadores envolvidos na manutenção da obesidade, agravados quando esta é associada a sintomas depressivos.

Diversas áreas têm evidenciado a família como objeto de estudo a partir do reconhecimento de que ela desempenha um papel fundamental no desenvolvimento e manutenção da saúde e no equilíbrio emocional de seus membros (Simionato \& Oliveira, 2003). Cardoso e Féres-Carneiro (2008) constataram que a família é uma referência fundamental na vida das pessoas, no sentido de experiência, de felicidade, suporte e promoção de equilíbrio pessoal. Contudo, neste estudo, a família foi associada também a experiências de conflitos, preocupações, expectativas negativas e catastróficas, solidão e sofrimento. Dessa forma, constata-se que nem sempre a família exerce função de proteção e promoção de bem-estar.

O suporte social, mais especificamente o suporte familiar, é apontado como um dos principais atenuadores do efeito de eventos estressores na vida dos indivíduos (Baptista, 2005). 0 suporte familiar abarca questões relativas às complexas relações que se estabelecem entre os membros de uma família 
e relaciona-se aos aspectos psicológicos, como manifestação de carinho, atenção, diálogo, proximidade afetiva, liberdade, superproteção e independência existente entre os membros da família (Baptista, Souza \& Alves, 2008).

O suporte familiar também correlaciona-se com saúde mental (Souza \& Baptista, 2008), autoconceito (Rigotto, 2006), estilos parentais e sintomatologia depressiva (Santana, 2008), entre outros.

No que diz respeito à obesidade, Souza et al. (2005) identificaram o apoio familiar como uma variável importante na manutenção do peso, assim como o comportamento alimentar disfuncional, estresse e sentimentos negativos, em mulheres após tratamento nutricional. Observou-se que a ausência de apoio social e familiar são os principais aspectos que dificultam a perda de peso e a consequente manutenção dessa perda. Além disso, identificou-se que enquanto pacientes que percebem apoio familiar relatam bem-estar e motivação, as que não sentem apoio mencionam sentimentos de frustração.

Dornelles, Anton e Pizzinato (2014) considera que os arranjos e movimentos dinâmicos das estruturas sociais e familiares são aspectos fundamentais relacionados ao sobrepeso e à obesidade infantil. No funcionamento dos sistemas familiares com membros adultos obesos, Otto (2007) através de um estudo analítico transversal com pacientes atendidos pelo Programa de Pesquisa e Atendimento em Obesidade (PASSO) do Hospital Universitário de Brasília, e de estudo de caso realizado com duas famílias desses pacientes, corrobora com a literatura no que diz respeito à existência de fronteiras internas difusas e fronteiras externas rígidas; pouco lazer; forte relação entre alimentação e pertencimento familiar; obesidade considerada como único problema enfrentado pela família e, em consequência disso, negação e baixa expressão de conflitos.

Tendo em vista o papel da família no fenômeno da obesidade e outros transtornos alimentares, o objetivo deste estudo foi verificar as correlações da percepção do suporte familiar com a sintomatologia depressiva e ansiosa. Procurouse investigar o papel do suporte familiar para as pessoas que se submetem ao tratamento da obesidade. Trabalhou-se com as hipóteses de que: $\mathrm{H} 1$ : os escores de ansiedade e depressão estão positivamente relacionados; $\mathrm{H} 2$ : quanto maior o escore de depressão, menor o suporte familiar percebido; e H3: quanto maior o escore de ansiedade, menor o suporte familiar percebido.

Apesar de diversos estudos investigarem o ambiente familiar de pessoas com obesidade (Otto, 2007), em uma revisão sistemática, utilizando as bases de dados da Literatura Latino-Americana e do Caribe em Ciências da Saúde (LILACS) e do Scientific Eletronic Libray Online (Scielo) com os termos "suporte familiar" e "família", cruzados com a palavra-chave "obesidade", foram encontrados poucos estudos que tratassem do tema, apenas um na LILACS, que possuía relação direta com a temática, os demais não tiveram como foco de estudo a família. Ressalta-se ainda que quando cruzadas as palavras "Obesidade", "Suporte Familiar", "Ansiedade" e "Depressão", não foi encontrado nenhum estudo nos últimos cinco anos.

Ratifica-se a importância de estudar tais construtos, possibilitando ampliar a compreensão das condições que interferem no tratamento das pessoas com obesidade, no intuito de criar alternativas de intervenção, bem como a proposição de ações estratégicas na área de saúde que busquem atender as demandas desse público.

\section{MÉTODO}

\section{PARTICIPANTES}

Participaram do estudo 39 pacientes, do sexo feminino, acima de 18 anos, que estavam em atendimento endocrinológico e nutricional no ambulatório de obesidade adulta do Instituto de Saúde Elpídio de Almeida (ISEA), localizado na cidade de Campina Grande, Paraíba. As participantes foram selecionadas por conveniência. Como critério de exclusão da amostra foram desconsiderados os pacientes que apresentavam alguma limitação de ordem cognitiva ou estado alterado de consciência devido o uso de substâncias químicas. Não foi possível ter acesso a nenhum paciente do sexo masculino, pois além dos usuários do serviço serem predominantemente do sexo feminino, no período de coleta não foi encontrado nenhum paciente do sexo masculino em tratamento com a equipe.

As participantes possuem em média 41 anos de idade (DP $=10,74)$. A Tabela 1 apresenta os dados socioeconômicos das participantes em termos de frequência e porcentagem. Do total da amostra, $40,0 \%$ possui o ensino fundamental, destas apenas a metade $(20,0 \%)$ possui o ensino fundamental completo. No tocante à religião, a maior frequência encontrada foi para a religião católica $(40,0 \%)$. No quesito renda familiar, há uma predominância de participantes que possuem renda entre um e dois salários mínimos $(60,0 \%)$. A maior parte das respondentes é casada (52,5\%). No que se refere ao tempo de tratamento, $40,0 \%$ estão em tratamento há cerca de dois anos. Quanto ao histórico familiar $87,5 \%$ das participantes afirmou ter algum familiar (pai, mãe, irmãos, tios, entre outros) com o mesmo histórico de obesidade. 
Tabela 1. Dados Socioeconômicos das Participantes $(n=39)$

\begin{tabular}{l|lll}
\hline Variáveis & Níveis & $F$ & $\%$ \\
\hline Escolaridade & Fundamental & 16 & $40,0 \%$ \\
& Médio & 14 & $35,0 \%$ \\
& Superior & 9 & $22,5 \%$ \\
\hline Religião & Sem religião & 1 & $2,5 \%$ \\
& Católica & 16 & $40,0 \%$ \\
& Protestante & 15 & $37,5 \%$ \\
& Outras & 7 & $17,5 \%$ \\
\hline Estado civil & Casado & 21 & $52,5 \%$ \\
& Solteiro & 13 & $32,5 \%$ \\
& Separado/divorciado & 3 & $7,5 \%$ \\
& Outros & 2 & $5,0 \%$ \\
\hline Renda & Menos de um salário & 4 & $10,0 \%$ \\
& Um e dois salários & 24 & $60,0 \%$ \\
& Três e quatro salários & 8 & $20,0 \%$ \\
& Cinco salários ou mais & 1 & $2,5 \%$ \\
& Não respondeu & 2 & $5,0 \%$ \\
\cline { 2 - 4 } Tempo no projeto & Menos de um ano & 10 & $25,0 \%$ \\
& Um - dois anos & 10 & $25,0 \%$ \\
& Dois- três anos & 16 & $40,0 \%$ \\
& Acima de três anos & 3 & $7,5 \%$ \\
\hline Histórico familiar & Sim & 35 & $87,5 \%$ \\
& Não & 4 & $10,0 \%$ \\
\hline
\end{tabular}

\section{INSTRUMENTOS}

Foram utilizados três instrumentos de avaliação para a coleta dos dados, além de uma ficha sociodemográfica:

1) Ficha sociodemográfica: elaborado pelos próprios pesquisadores, composto de perguntas, tais como: sexo, idade, estado civil, escolaridade, religião, renda mensal da família, além de questionamentos sobre histórico familiar de obesidade.

2) Inventário de Depressão de Beck - BDI (Cunha, 2001): instrumento universalmente empregado na clínica e em levantamentos com a população em geral, composto de 21 itens, utilizados para aferir a intensidade da depressão. 0 BDI é constituído por subescalas que avaliam os componentes cognitivos-afetivos, comportamentais e queixas somáticas. A escala é graduada com afirmações de zero a três. Seus itens incluem: tristeza, pessimismo, sentimento de fracasso, insatisfação, culpa, punição, autoaversão, autoacusações, ideias suicidas, choro, irritabilidade, retraimento social, mudança na autoimagem, dificuldade de trabalhar, insônia, fatigabilidade, perda de apetite, perda de peso, preocupações somáticas e perda da libido. $\mathrm{O}$ BDI mostrou-se um instrumento de alta confiabilidade.

3) Inventário de Ansiedade de Beck - BAI (Cunha, 2001): é constituído por 21 itens, compostos de afirmações descritivas de sintomas de ansiedade. Estas afirmações são avaliadas pelo sujeito numa escala de quatro pontos, refletindo níveis de gravidade crescente de cada sintoma: 0) Absolu- tamente não; 1) Levemente: não me incomodou muito; 2) Moderadamente: foi muito desagradável, mas pude suportar; 3) Gravemente: dificilmente pude suportar. Os itens do BAI se referem a: dormência ou formigamento, sensação de calor, tremores nas pernas, incapacidade de relaxar, medo que aconteça o pior, atordoamento ou tontura, palpitação ou aceleração do coração, sem equilíbrio, aterrorizado, nervoso, sensação de sufocação, tremores nas mãos, trêmulo, medo de perder o controle, dificuldade de respirar, medo de morrer, assustado, indigestão ou desconforto abdominal, sensação de desmaio, rosto afogueado e suor (não devido ao calor). 0 BAI mostrou adequadas propriedades psicométricas de acordo com suas pesquisas originais.

4) Inventário de Percepção de Suporte Familiar - IPSF (Baptista, 2010): foi desenvolvido com o objetivo de construir, validar e fornecer maneiras de mensurar o construto de suporte familiar no Brasil. Os dados estão dispostos em uma escala tipo Likert de três pontos, com as afirmativas: "sempre ou quase sempre", "às vezes" e "quase nunca ou nunca". 0 inventário é dividido em três dimensões, quais sejam: 1) Afetivo-consistente: composto por 21 itens que se referem às relações afetivas positivas intrafamiliares e aos papéis e regras dos integrantes da família. As perguntas são alusivas a interesse, proximidade, acolhimento, comunicação, interação, respeito, empatia, clareza nas regras intrafamiliares, consistência de comportamentos e verbalizações e habilidades na resolução de problemas; 2) Adaptação familiar: composto por 13 itens, esse fator é uma percepção geral de um baixo ou inadequado suporte familiar, as perguntas referem-se a sentimentos e comportamentos negativos em relação à família, como: raiva, isolamento, incompreensão, exclusão, não pertencimento, vergonha, irritação, relações agressivas, competição, interesse e etc.; e 3) Autonomia: composto por oito itens, agrupa a percepção de confiança, liberdade e privacidade que o indivíduo tem da sua família. Os resultados propõem que quanto maior o escore obtido, maior o suporte familiar percebido pelo sujeito. O IPSF mostrou adequadas propriedades psicométricas, tornando confiável a sua aplicação.

\section{PROCEDIMENTOS DE COLETA}

Conforme as exigências solicitadas pela Resolução 466/12 do Conselho Nacional de Saúde - CNS, este estudo foi submetido e aprovado pelo Comitê de Ética em Pesquisa da Universidade Estadual da Paraíba (CAAE 32203814.1.0000.5187). Após a aprovação, a coleta de dados foi executada ao longo de dois meses ininterruptos. As participantes consentiram sua participação voluntária na pesquisa e, após as devidas explicações, assinaram o Termo de Consentimento Livre e Esclarecido (TCLE). O tempo de 


\section{\# LF PSICOLOGIA}

Nathália Gomes da Silva, José Ronieri Morais Batista, Melyssa Kellyane Cavalcanti Galdino e Sibelle Maria Martins de Barros. aplicação foi de aproximadamente 35 minutos. Os pacientes foram abordados enquanto esperavam por atendimento médico na sala de espera.

\section{PROCEDIMENTOS DE ANÁLISE}

Os dados foram analisados a partir da estatística descritiva, para caracterização socioeconômica e análise da incidência e prevalência da ansiedade e depressão (frequência, média, porcentagem e desvio padrão). Para as análises correlacionais, utilizou-se a estatística paramétrica de Pearson. A significância estatística adotada foi de $5 \%(0,05)$. Para isso usou-se o Pacote Estatístico Para as Ciências Sociais SPSS, versão 20.0 .

\section{RESULTADOS E DISCUSSÃO}

Para a análise dos resultados, inicialmente será apresentada a distribuição dos participantes de acordo com a pontuação média nos escores do BDI, BAI e IPSF. Essa distribuição permitiu verificar como a amostra se caracteriza de forma geral, em termos dos sintomas depressivos, ansiosos e do suporte familiar percebido. Em seguida, foi calculado o coeficiente de correlação de Pearson para análise das correlações entre os escores do BDI, $\neg$ BAI e IPSF. Por fim, também foram analisadas as correlações entre o BDI, BAI e cada um dos três fatores do IPSF.

A tabela 2 mostra a distribuição dos participantes de acordo com as médias no escore total do BDI, do BAI e do IPSF, assim como nas dimensões do IPSF. No BDI a média dos participantes foi de 12,35 ( $D P=7,98)$, sendo 0 o mínimo atingido e 35 o máximo. Segundo dados normativos, o ponto de corte utilizado para rastreamento de depressão em estudos com população não clínica é de até 20 pontos (Cunha, 2001), o que caracteriza os participantes com ausência de sintomas depressivos. No BAI a média foi $12,58(\mathrm{DP}=9,73)$ variando entre 0 , o mínimo, a 41 , resultado máximo. Em amostras não clínicas, o escore abaixo de 20 pontos também é considerado como indicativo da inexistência de ansiedade clinicamente significativa (Cunha, 2001). Os escores do IPSF, ao serem comparados com os resultados do manual (Baptista, 2010) apontaram que, na dimensão Afetivo-Consistente, os participantes obtiveram uma classificação média-alta, com média de 30,38 (DP = 8,12); no fator Adaptação Familiar a média foi de 21,35 (DP = 3,69), classificado como médiabaixa; no tocante ao fator Autonomia Familiar a média foi de $14,58(\mathrm{DP}=1,88$ ) classificada como média-alta; no total do IPSF os participantes obtiveram uma média de 66,33 (DP = $11,29)$ atingindo assim uma classificação média-alta.

Foi realizada uma análise correlacional entre os dados sociodemográficos e os escores de depressão, ansiedade e
Tabela 2Distribuição da Amostra de Acordo Com a Pontuação no $B D I, B A l$ e Dimensões do IPSF $(n=39)$

\begin{tabular}{ccccc}
\hline & Mínimo & Máximo & Média & Desvio Padrão \\
\hline BDI & 0 & 35 & 12,35 & 7,98 \\
BAI & 0 & 41 & 12,58 & 9,73 \\
IPSF & 35 & 84 & 66,33 & 11,29 \\
Afetivo-consistente & 7 & 42 & 30,38 & 8,12 \\
Adaptaçãofamiliar & 10 & 26 & 21,35 & 3,69 \\
Autonomia & 10 & 16 & 14,58 & 1,88 \\
\hline
\end{tabular}

de suporte familiar. Das variáveis analisadas apenas o grau de escolaridade apresentou uma correlação significativa entre o escore do $\mathrm{BDI}$, de forma que quando maior o nível de escolaridade, menor era o escore de depressão $\left(r=-0,448 ; r^{2}=\right.$ 20,07\%; $p<0,05)$.

Além das análises correlacionais foram realizados testes de comparação grupal (teste $t$ ) entre as médias dos escores depressão, ansiedade e de suporte familiar em função do estado civil (casado ou solteiro). Foram encontradas diferenças significativas apenas para o escore de depressão $(t=2,095 ; p$ $<0,05)$, onde o grupo de pessoas que eram casadas apresentavam uma maior média de depressão $(M=13,15$; $D P=7,80)$ em relação ao grupo de pessoas solteiras $(M=8,38$; $D P=$ $5,27)$. A normalidade das variáveis foi verificada através do teste de Komolgorov-Smirnov, onde foi obtido $z=0,159$ ( $p=$ n.s.) para o grupo dos participantes casados e $z=0,221$ ( $p=$ n.s.) para o grupo dos solteiros.

Também foi realizado um teste $t$ de Student para as médias dos escores de depressão, ansiedade e suporte familiar em função da religião dos participantes. Foi encontrada uma diferença estatisticamente significativa entre o grupo dos católicos e dos protestantes $(t=2,237 ; p<0,05)$, de forma que os católicos ( $M=15,28$; DP = 11,62) apresentam maiores índices de ansiedade em relação ao grupo dos protestantes (M $=8,25 ; \mathrm{DP}=5,03)$. A normalidade das variáveis foi verificada por meio do teste de Komolgorov-Smirnov, onde foi obtido $z=$ 0,145 ( $p=$ n.s.) para o grupo dos católicos e $z=0,207$ ( $p=$ n.s.) para o grupo dos protestantes.

Com o objetivo de se verificar a relação entre sintomatologia depressiva e ansiosa e a percepção do suporte familiar, foi realizada uma análise correlacional entre os escores das três variáveis, como pode se verificar na Tabela 3. Foram verificadas as normalidades das variáveis em questão através do teste de Komolgorov-Smirnov, onde foi observado que cada um dos três escores utilizados possuíam distribuição normal (BDI: $Z=0,895 ; p=$ n.s.; BAI: $Z=1,110 ; p=$ n.s.; IPSF: $Z=$ 0,$899 ; p=n . s$.). Um teste de correlação de Pearson (bicaudal) detectou uma correlação positiva significativa $(p<0,01)$ de 0,431 entre os escores de Depressão e Ansiedade, que explica $18,57 \%$ da variância observada (corroborando a previsão 


Tabela 3. Coeficiente de Correlação Entre
Percepção do Suporte Familiare
\begin{tabular}{l|ll} 
Sintomatologia Depressivae Ansiosa (n = 39) \\
\hline & BDI & BAI \\
\hline BDI & - & \\
BAI & $0,431 * \star$ & - \\
IPSF & $-0,361 *$ & $-0,456$ ** \\
\hline * $<<0,05 ; * \star p<0,01$ &
\end{tabular}

da Hipótese H1). De modo que, quanto maior o grau de depressão que as pacientes apresentavam maior também era o nível de ansiedade. No que concerne à análise correlacional do IPSF e do BDI, foi encontrada uma correlação negativa significativa $(p<0,05)$ de $-0,361$, que explica $13,03 \%$ da variância observada. No IPSF e no BAI também foi encontrada uma correlação negativa significativa $(p<0,01)$ de $-0,456$, que explica $20,79 \%$ da variância observada. Desta forma, na medida em que os participantes percebiam o Suporte Familiar como adequado menores eram as pontuações obtidas no BDI e no BAI (corroborando a previsão das hipóteses H2 e H3). Ressalta-se que, devido ao baixo número de participantes na amostra, novas coletas poderiam auxiliar na comprovação de tais resultados.

Após a análise correlacional dos escores totais, realizouse também uma análise correlacional entre as dimensões do IPSF e o escore total do BDI e do BAI, visando verificar a magnitude das dimensões e suas respectivas relações sobre os sintomas de depressão e de ansiedade.

Conforme pode ser visualizado na Tabela 4, todas as dimensões do IPSF se correlacionaram negativamente com o BDI e com o BAI. No que diz respeito ao BDI, apenas o fator 2, Adaptação familiar, se correlacionou significativamente ( $p<$ 0,01 ) atingindo $-0,519$ que explica $26,93 \%$ da variância observada. Dessa forma, quanto menor o escore do fator adaptação familiar, maiores os escores de depressão. Já para o escore do BAl, dois fatores apresentaram correlações negativas e significativas. 0 fator 1 , afetivo-consistente, teve correlação de $-0,349$ ( $p<0,05)$, que explica $12,18 \%$ da variância observada; e o fator 2, adaptação-familiar, teve correlação de $-0,649(p<0,01)$, que explica $42,12 \%$ da variância observada. Assim, quanto maiores os escores observados nos fatores de adaptação familiar e afetivo-consistente, menor foi o escore no inventário de ansiedade.

\section{DISCUSSÃO}

Associações entre depressão e escolaridade em diferentes populações são frequentes na literatura (Cunha, Bastos, \& Duca, 2012; Furlanetto, Moral, Gonçalves, Rodrigues, \& Jaco-

\begin{tabular}{|c|c|c|}
\hline Dimensões do IPSF & BDI & BAI \\
\hline Afetivo- consistente & $-0,281$ & $-0,349 *$ \\
\hline Adaptação familiar & $-0,519 * \star$ & $-0,649 * *$ \\
\hline Autonomia & $-0,163$ & $-0,167$ \\
\hline
\end{tabular}

mino, 2006; Ruschil, et al., 2007;; Sousa, et al., 2010;). Já o casamento comumente é visto como fator de proteção para a depressão. No entanto, esse dado se aplica para os homens, mas não para as mulheres (Santos \& Kassouf, 2007), o que corroborou com o resultado encontrado no presente artigo, o qual sua amostra é composta apenas de mulheres. Correlações entre catolicismo e ansiedade não têm sido descritas na literatura, não foram encontrados estudos que abordassem essa relação.

No tocante à relação entre ansiedade e depressão, foram encontrados dados que corroboram a literatura sobre o tema. Estes construtos são considerados independentes, mas é reconhecida uma associação entre eles, tanto do ponto de vista da saúde mental como do ponto de vista estatístico (Pais-Ribeiro, Honrado, \& Leal, 2004). Associações entre depressão e ansiedade em pacientes obesos foram confirmadas pelo estudo de Soares (2009) que encontrou sintomas de ansiedade em $71,5 \%$ dos indivíduos diagnosticados com obesidade e $38,5 \%$ de depressão, tendo verificado em $33,8 \%$ dos pacientes a existência de depressão e ansiedade simultaneamente.

No presente estudo, as correlações entre a ansiedade e suporte familiar, bem como entre depressão e suporte familiar, foram negativas, significativas e moderadas. Este resultado já era previsto, uma vez que na literatura valores altos de depressão e de ansiedade comumente estão associados com valores baixos de percepção do suporte familiar (Baptista, 2005).

Atualmente, a literatura aborda a importância do suporte familiar e social para amostras específicas e sua relação com aspectos psicológicos e sociais. Estudos realizados por Santana (2008) e Lemos, Baptista e Carneiro (2011), indicaram a importância do suporte familiar na sintomatologia depressiva. Nesse sentido, os estudos mostram que quanto maior a percepção de autonomia, de afetividade e de adaptação, dentre outras características do suporte familiar, menor o número de sintomas depressivos e de crenças irracionais. Além disso, resultados encontrados por Bueno (2009) indicaram que níveis altos de ansiedade-traço/estado estão associados a níveis baixos de percepção do suporte familiar. 
Em uma pesquisa, Baptista e Oliveira (2004) procuraram avaliar a correlação existente entre sintomatologia depressiva e as percepções de suporte familiar. Os resultados obtidos indicaram que o suporte familiar esteve avaliado negativamente no grupo que possuía sintomatologia depressiva, apontando que quanto maior a sintomatologia depressiva, mais negativa era a percepção do suporte familiar recebido. Os autores chamaram atenção ainda para o fato de que os sintomas depressivos também podem estar envolvidos na percepção do suporte familiar, já que indivíduos com depressão tendem a perceber o seu suporte familiar como mais supercontrolador e fornecendo menores taxas de carinho.

De acordo com Campos (2007), o efeito principal do suporte acontece à medida que o receptor o percebe como acessível e satisfatório, e esta percepção é decorrente do fato de o indivíduo sentir-se valorizado, amado, reconhecido, compreendido, cuidado e protegido, assim como pertencente a uma rede de informações e recursos que com ele são partiIhados. As consequências positivas, que contribuem para redução do estresse, aumento da autoestima e bem-estar psicológico, advêm da percepção que o indivíduo tem de sentir-se capaz de enfrentar o ambiente.

Por isso, faz-se necessário considerar a possibilidade de existirem outros fatores que influenciaram nesses resultados, entre eles: as condições socioeconômicas das participantes, o uso de medicamentos (ansiolíticos e antidepressivos) que afeta a maneira pela qual o sujeito interage com a família, o suporte oferecido pelo próprio serviço, entre outros.

Cumpre ressaltar que, para o modelo cognitivo, o modo como as pessoas se sentem está relacionado à forma como elas interpretam e pensam sobre as situações, isto é, não é a situação por si só que determina como elas se sentem; a percepção da situação serve como mediadora da resposta emocional do sujeito (Beck, 1997).

No que tange às dimensões do IPSF, que se correlacionaram negativamente com o $\mathrm{BDI}$ e com o BAI, foi encontrado resultado semelhante por Lemos, Batista e Carneiro (2011). No referido estudo, a magnitude correlacional entre o IPSF com o $\mathrm{BDI}$ foi fraca nos fatores afetivo-consistente e autonomia familiar, e moderada no fator adaptação familiar. Dessa forma, no presente trabalho, chama-se atenção para o fator adaptação familiar, que foi significativo tanto em relação à sintomatologia depressiva quanto à ansiosa, o que pode indicar sua forte influência na determinação de uma percepção adequada do suporte familiar para pacientes obesos. 0 fator em questão é composto de afirmações voltadas para sentimentos e comportamentos negativos em relação à família, tais como: "Eu me sinto como um estranho na minha família"; "Viver com minha família é desagradável". Tais afirmações denotam diretamente a sensação de não se perceber como um membro da família. Esse posicionamento sugere que o sentimento de pertença talvez seja mais importante do que demonstrar carinho através de palavras ou do que ter privacidade de uma forma geral.

A ênfase na necessidade de se sentir aceito pode ser desdobramento da pressão social sofrida por pessoas obesas que fogem do padrão corpóreo tomado como ideal por parte da sociedade. Tipicamente são atribuídas às pessoas obesas as características de gulosas, preguiçosas, desleixadas e sem força de vontade (Felippe et al., 2004). Ter um lugar onde se sentem seguras e acolhidas torna-se fundamental. Este resultado indica a importância do fator adaptação familiar, ao ponto de que, quando não é percebido como ideal, correlaciona-se com um aumento nos escores de ansiedade e depressão.

A importância da adaptação familiar é reforçada pela literatura na qual os conflitos e elementos abarcados pela segunda dimensão do IPSF são um importante preditor de funcionamento familiar disfuncional e consequentemente a percepção de ausência de suporte (Baptista, 2007). Ainda segundo o referido autor, a solução de problemas, a coesão/vínculos, também estariam especificamente relacionadas com a habilidade da família em resolver e apoiar os membros em situações-problema.

Nesse sentido, entende-se que a família contribui no fortalecimento da visão de si mesmo no que tange ao domínio emocional. A intervenção de familiares, ao expressar solidariedade e oferecer amor, esperança e conforto, minimiza sentimentos de raiva e culpa, assim como sintomas de ansiedade e depressão (Campos, 2007). Em vista disso, faz-se necessário a construção de uma percepção de apoio familiar para pacientes obesos.

O trabalho do psicólogo com estes usuários torna-se fundamental. Sugere-se que as intervenções devam abordar as crenças construídas no ambiente social e cultural, assim como as questões advindas da história de vida de cada um. Nesse processo, informações são trocadas, são construídos novos saberes e vínculos, e estratégias são criadas para o enfretamento da obesidade e melhoria da qualidade de vida.

Em sua maioria, os resultados demonstraram a efetiva importância do suporte social, principalmente do suporte familiar, que se oferecido/percebido como adequado pelo indivíduo, pode se apresentar como um fator de proteção no processo de controle de peso, especialmente no que diz respeito à adesão ao tratamento. Já o suporte familiar insuficiente, por sua vez, pode contribuir para o comprometimento da saúde (Palmeira, Garrido, \& Resumo, 2016).

Possíveis associações entre depressão ansiedade e compulsão alimentar têm sido frequentemente relatadas na litera- 
tura nos últimos anos, especialmente entre aqueles pacientes candidatos à cirurgia bariátrica (Krukowski, Friedman, \& Applegate, 2010; Machado, Zilberstein, Cecconello, \& Monteiro, 2008; Oliveira \& Yoshida, 2009; Petribu et al., 2006; Wedin et al., 2012). Porém, nos estudos realizados no Brasil, são encontradas divergências com relação à presença de transtornos psicológicos, especialmente ansiedade e depressão (Ribeiro, Giampietro, Belarmino, \& Salgado, 2016). A maioria dos estudos conduzidos nessa área possui um baixo número de participantes na amostra, o que dificulta generalização dos achados (Mota, Costa, \& Almeida, 2014; Tae et al., 2014). Não foram encontrados até o momento estudos que relacionassem tais sintomatologias ao suporte familiar.

Cumpre ressaltar que obesos que não estão em tratamento para obesidade podem ter resultados diferentes dos encontrados nesta pesquisa, por isso, pressupõe-se relevante replicar esta análise utilizando os dois grupos: de pacientes obesos em tratamento e pacientes obesos que não se encontram em tratamento.

Por se tratar de uma amostra reduzida, os dados apresentados neste trabalho ainda são iniciais. Dessa forma, faz-se necessário o desenvolvimento de outros estudos que busquem avaliar a importância do suporte familiar para os riscos de desenvolver, assim como de amenizar sintomas de depressão e de ansiedade em pacientes em tratamento para obesidade, a fim de potencializar os processos de intervenções, atendendo as legítimas necessidades deste público.

\section{CONTRIBUIÇÃO DE CADA AUTOR}

N.G.S. foi responsável pela execução da pesquisa, redação inicial do artigo, revisão e edição do texto. J.R.M.B. foi responsável pela supervisão, execução das análises dos dados, revisão e edição do texto. M.K.C.G. foi responsável pelas contribuições referentes aos aspectos teóricos da Terapia Cognitiva, revisão e edição do texto. S.M.M.B. foi responsável pela supervisão, pelas contribuições referentes aos aspectos teóricos do Suporte Familiar, revisão e edição do texto.

\section{DECLARAÇÃO DE CONFLITOS DE INTERESSES}

Os autores declaram que não há conflitos de interesses no presente artigo.

\section{REFERENNCIAS}

ABESO. Associação Brasileira para o Estudo da Obesidade e da Síndrome Metabólica -ABESO (2002). Métodos de avaliação de obesidade e alguns dados epidemiológicos. Recuperado de http://www.abeso.org.br/pagina/194/ metodos-de-avaliacao-de-obesidade-e-alguns-dadosepidemiologicos.html

Ades, L., \& Kerbauy, R. R. (2002). Obesidade: realidade e indignações. Psicologia USP, 13(1), 197-216. http://dx.doi.org/ 10.1590/S0103-65642002000100010

Baptista, M. N. (2005). Desenvolvimento do inventário de percepção de suporte familiar (IPSF): Estudos psicométricos preliminares. Psico-USF, 10(1), 11-19. http://dx.doi.org/ 10.1590/S1413-82712005000100003

Baptista, M. N. (2007). Inventário de percepção de suporte familiar (IPSF): Estudo componencial em duas configurações. Psicologia Ciência e Profissão, 27(3), 496-509. http:// dx.doi.org/10.1590/S1414-98932007000300010

Baptista, M. N. (2010). IPSF: Inventário de percepção de suporte familiar [Manual]. São Paulo: Vetor.

Baptista, M. N., Cardoso, H. F., \& Gomes, J. O. (2012). Intergeracionalidade familiar. Em Baptista, M. N., \& Teodoro, M. L. M. (Orgs.). Psicologia de família: Teoria, avaliação e intervenção (pp. 16-26). Porto Alegre: Artmed.

Baptista, M. N., \& Oliveira, A. A. (2004). Sintomatologia de depressão e suporte familiar em adolescentes: um estudo de correlação. Journal of Human Growth and Development, 14(3), 53-59. http://dx.doi.org/10.7322/jhgd.40168

Baptista, M. N., Souza, M. S., \& Alves, G. A. S. (2008). Evidências de validade entre a Escala de Depressão (EDEP), o BDI e o Inventário de Percepção de Suporte Familiar (IPSF). Psico-USF, 13(2), 211-220. http://dx.doi.org/ $10.1590 /$ S1413-82712008000200008

Beck, J. S. (1997). História da terapia cognitiva. Em J. Beck (Ed). Terapia cognitiva: Teoria e prática (pp. 17-27). Porto Alegre, RS: Artmed.

Bueno, M. F. (2009). Avaliação da ansiedade e percepção do suporte familiar em hipertensos. (Dissertação de mestrado). Universidade São Francisco, Itatiba, SP.

Campos, E. P. (2007). Suporte social e família. Em J. Melo FiIho, \& M. Burd (Org.). Doença e família (pp. 141 -164). São Paulo: Casa do Psicólogo.

Cardoso, C. L. \& Féres-Carneiro, T. F. (2008). Sobre a família: Com a palavra, a comunidade. Estudos e Pesquisas em Psicologia, 8(2), 511-526.

Correia, T. I. G., \& Afonso, I. L. (2012). Obesidade e sintomatologia depressiva em estudantes do ensino superior. Pôster apresentado no $16^{\circ}$ Congresso Português de Obesidade, Lisboa, Portugal.

Coutinho, W. F. (1999). Consenso Latino Americano de Obesidade. Arquivos Brasileiros de Endocrinologia e Metabologia, 43(1), 21-67. http://dx.doi.org/10.1590/ S0004-27301999000100003

Coutinho W. (2007). Etiologia da obesidade. Revista ABESO, $7(30)$.

Cunha, A. J. (2001). Manual da Versão em Português das Escalas Beck [Manual]. São Paulo: Casa do Psicólogo. 
Cunha, R. V., Bastos, G. A. N., \& Duca, G. F. (2012). Prevalência de depressão e fatores associados em comunidade de baixa renda de Porto Alegre, Rio Grande do Sul. Revista Brasileira de Epidemiologia, 15(2), 346-54. http:// dx.doi.org/10.1590/S1415-790X2012000200012

Dornelles, A. D., Anton, M. C., \& Pizzinato, A. (2014). O papel da sociedade e da família na assistência ao sobrepeso e à obesidade infantil: Percepção de trabalhadores da saúde em diferentes níveis de atenção. Saúde e Sociedade, 23(4), 1275-1287. http://dx.doi.org/10.1590/S010412902014000400013

Felippe, F. M. L. Friedam., R., Alves, B. S., Cibeira, G. H., Surita, L. E., \& Tesche. C. (2004). Obesidade e mídia: O lado sutil da informação. Revista Acadêmica do Grupo Comunicacional de São Bernardo, 1(2), 1-5.

Furlanetto, L., Moral, J. A. G. D., Gonçalves, A. H. B., Rodrigues, K., \& Jacomino, M. E. M. L. P. (2006). Diagnosticando depressão em pacientes internados com doenças hematológicas: Prevalência e sintomas associados. Jornal Brasileiro de Psiquiatria, 55(2), 96-101. http://dx.doi.org/ 10.1590/S0047-20852006000200001

Gold, M. S., Oppenheim, H. C., \& Frost-Pineda, K. (2006). Obesity and substance use disorders. In S. L. McElroy, D. B. Allison, G. A. Bray(Ed.) Obesity and mental disorders (pp. 103-122). Taylor \& Francis Group: London.

Krukowski, R. A., Friedman, K. E., \& Applegate, K. L. (2010). The utility of the beck depression inventory in a bariatric surgery population. Obesity Surgery, 20(4), 426-31. http:// dx.doi.org/10.1007/s11695-008-9717-2

Lemos, V. A., Baptista, M. N., \& Carneiro, A. M. (2011). Suporte familiar, crenças irracionais e sintomatologia depressiva em estudantes universitários. Psicologia: Ciência e Profissão, 31(1), 20-29. http://dx.doi.org/10.1590/S141498932011000100003

Machado, C. E., Zilberstein, B., Cecconello, I., \& Monteiro M. (2008). Compulsão alimentar antes e após a cirurgia bariátrica. ABCD. Arquivos Brasileiros de Cirurgia. Digestiva, 21(4), 185-91. http://dx.doi.org/10.1590/S010267202008000400007

Matos, M. I. R., Aranha, L. S. Faria, A. N., Ferreira, S. R. G., Bacaltchuckc, J., \& Zanella, M. T. (2002). Binge eating disorder, anxiety, depression and body image in grade III obesity patients. Revista Brasileira de Psiquiatria, 24(4), 165-169. http://dx.doi.org/10.1590/S151644462002000400004

Ministério da Saúde, Secretaria de Vigilância em Saúde, Departamento de Vigilância de Doenças e Agravos não Transmissíveis e Promoção da Saúde (2015). Vigilância de Fatores de Risco e Proteção para Doenças Crônicas por Inquérito Telefônico - VIGITEL BRASIL. Recuperado de http://bvsms.saude.gov.br/bvs/publicacoes/vigitel_brasil_2014.pdf
Moraes, A. L., Almeida, E. C., \& Souza. L. B. (2013). Percepções de obesos deprimidos sobre os fatores envolvidos na manutenção da sua obesidade: Investigação numa unidade do Programa Saúde da Família no município do Rio de Janeiro. Physis Revista de Saúde Coletiva 23(2),553572. 3000200012

Mota, D. C. L., Costa, T. M. B., \& Almeida, S. S. (2014). Imagem corporal, ansiedade e depressão em mulheres submetidas à cirurgia bariátrica. Psicologia: Teoria e Prática, 16(3), 100-13.

Oliveira, J. H. A., \& Yoshida, E. M. P. (2009). Avaliação psicológica de obesos grau III antes e depois de cirurgia bariátrica. Psicologia: Reflexão e Crítica, 22(1), 12-9. http:// dx.doi.org/10.1590/S0102-79722009000100003

Otto, A. F. N. (2007). Obesidade e Transtorno da Compulsão alimentar Periódica: um estudo sobre a dinâmica familiar (Dissertação de mestrado). Universidade Católica de Brasília, Brasília, DF. Recuperado de http://www.bdtd.ucb.br/ tede/tde_busca/arquivo.php?codArquivo $=923$

Pais-Ribeiro, J. L., Honrado, A., \& Leal, I. (2004). Contribuição para o estudo da adaptação portuguesa das escalas de ansiedade, depressão e stress (EADS) de 21 itens de Lovibond e Lovibond. Psicologia, Saúde \& Doenças, V(2), 229239.

Palmeira, C. S., Garrido, L. M. M., \& Resumo, P. S. (2016). Fatores intervenientes na adesão ao tratamento da obesidade. Ciencia y Enfermería, 22(1), 11-22. http://dx.doi.org/ $10.4067 /$ S0717-95532016000100002

Petribu, K., Ribeiro, E. S., Oliveira, F. M. de, Braz, C.I., Gomes, M. L. M., Araujo, D. E. D., \& Ferreira, M. N. L. (2006). Transtorno da compulsão alimentar periódica em uma população de obesos mórbidos candidatos a cirurgia bariátrica do Hospital Universitário Oswaldo Cruz, em Recife-PE. Arquivos Brasileiros de Endocrinologia Metabólica, 50(5), 901-08. http://dx.doi.org/10.1590/S000427302006000500011

Ribeiro, G. A. N. A., Giampietro, H. B., Belarmino, L. B., \& Salgado Jr., W. Jr. (2016). Psychological profile of patients eligible for bariatric surgery. ABCD. Arquivos Brasileiros de Cirurgia Digestiva, 29(Supl. 1), 27-30. http://dx.doi.org/ 10.1590/0102-6720201600S10008

Riggotto, D. M. (2006). Evidências de validade entre suporte familiar, suporte social e autoconceito (Dissertação de mestrado). Universidade São Francisco, Itatiba, SP.

Ruschil, G. E. C., Sun, S. Y., Mattar, R., Chambô Filho, A., Zandonade, E., \& Lima, V. J. (2007). Aspectos epidemiológicos da depressão pós-parto em amostra brasileira. Revista de Psiquiatria do Rio Grande do Sul, 29(3), 274$280 . \quad \mathrm{http} / / / \mathrm{dx}$.doi.org/10.1590/S010181082007000300006 
Santana, P. R. (2008). Suporte Familiar, Estilos Parentais e Sintomatologia Depressiva: Um Estudo Correlacional (Dissertação de mestrado). Universidade São Francisco, Itatiba, SP.

Santos, M. J., \& Kassouf, A. L. (2007). Uma investigação dos determinantes socioeconômicos da depressão mental no Brasil com ênfase nos efeitos da educação. Economia Aplicada, 11(1), 5-26. https://dx.doi.org/10.1590/S141380502007000100001

Saueressig, M. R., \& Berleze, K. J. (2010). Análise de influências em dietas de emagrecimento. PSICO, 41(2), 231-238.

Simionato, M. A. W., \& Oliveira, R. G. (2003). Funções e transformações da família ao longo da história. Em Anais, I Encontro Paranaense de Psicopedagogia. Maringá, PR: Associação Brasileira de Psicopedagogia.

Soares, A. S. B. (2009). Qualidade de vida, depressão e ansiedade em indivíduos que procuram tratamento para a obesidade (Dissertação de mestrado). Universidade Fernando Pessoa, Porto. Recuperado de http://bdigital.ufp.pt/handle/ $10284 / 1569$

Sousa, M., Nunes, A., Guimarães, A. I., Cabrita, J. M., Cavadas, L. F., \& Alves, N. F. (2010). Depressão em idosos: Prevalência e factores associados. Revista Portuguesa de Medicina Geral e Familiar, 26(4), 384-391.

Souza, J. M. B., Castro, M. M., Maia, E. M. C., Ribeiro, A. N., Almondes, K. M., \& Silva, N. G. (2005). Obesidade e tratamento: Desafio comportamental e social. Revista Brasileira de Terapias Cognitivas, 1(1), 59-67.

Souza, M. S., \& Baptista, M. N. (2008). Associações entre suporte familiar e saúde mental. Psicologia Argumento, 26(54), 207-215.
Tae, B., Pelaggi, E. R., Moreira, J. G., Waisberg, J., Matos, L. L., \& D’Elia, G. (2014). O impacto da cirurgia bariátrica nos sintomas depressivos e ansiosos, comportamento bulímico e na qualidade de vida. Revista do Colégio Brasileiro de Cirurgiões, 41(3), 155-160. http://dx.doi.org/10.1590/ S0100-69912014000300004

Vasques, F., Martins, F., \& Azevedo, A. (2004). Aspectos psiquiátricos do tratamento da obesidade. Archives of Clinical Psychiatry (São Paulo), 31(4), 195-198. http:// dx.doi.org/10.1590/S0101-60832004000400013

Vargas, V. (2012). Elementos para pensar a obesidade, a depressão e a ansiedade. Trabalho de Conclusão de Curso de Pós-graduação, Universidade Regional do Noroeste do Estado do Rio Grande do Sul, ljuí. Recuperado de http://bibliodigital.unijui.edu.br:8080/xmlui/handle/ $123456789 / 1226$ ?show =full

Wedin, S., Byrne, K., Morgan, K., Lepage, M., Goldman, R., Crowley N., \& Borckardt, J. J. (2012). Presurgical weight is associated with pain, functional impairment, and anxiety among gastric bypass surgery patients. Pain Research and Treatment, 2012. http://doi.org/10.1155/2012/412174

Recebido em: 06/06/2016 Primeira decisão editorial em: 28/04/2017 Aceito em: 08/02/2018 